\title{
, Fundamental Ice Crystal Accretion Physics Studies
}

\begin{abstract}
Due to numerous engine power-loss events associated with high-altitude convective weather, ice accretion within an engine due to ice-crystal ingestion is being investigated. The National Aeronautics and Space Administration (NASA) and the National Research Council (NRC) of Canada are starting to examine the physical mechanisms of ice accretion on surfaces exposed to ice-crystal and mixed-phase conditions. In November 2010, two weeks of testing occurred at the NRC Research Altitude Facility utilizing a single wedge-type airfoil designed to facilitate fundamental studies while retaining critical features of a compressor stator blade or guide vane. The airfoil was placed in the NRC cascade wind tunnel for both aerodynamic and icing tests. Aerodynamic testing showed excellent agreement compared with CFD data on the icing pressure surface and allowed calculation of heat transfer coefficients at various airfoil locations. Icing tests were performed at Mach numbers of 0.2 to 0.3 , total pressures from 93 to $45 \mathrm{kPa}$, and total temperatures from 5 to $15^{\circ} \mathrm{C}$. Ice and liquid water contents ranged up to 20 and $3 \mathrm{~g} / \mathrm{m}^{3}$, respectively. The ice appeared well adhered to the surface in the lowest pressure tests $(45 \mathrm{kPa}$ ) and, in a particular case, showed continuous leadingedge ice growth to a thickness greater than 15 millimeters in 3 minutes. Such widespread deposits were not observed in the highest pressure tests, where the accretions were limited to a small area around the leading edge. The suction surface was typically ice-free in the tests at high pressure, but not at low pressure. The icing behavior at high and low pressure appeared to be correlated with the wet-bulb temperature, which was estimated to be above $0^{\circ} \mathrm{C}$ in tests at $93 \mathrm{kPa}$ and below $0^{\circ} \mathrm{C}$ in tests at lower pressure, the latter enhanced by more evaporative cooling of water. The authors believe that the large ice accretions observed in the low pressure tests would undoubtedly cause the aerodynamic performance of a compressor component such as a stator blade to degrade significantly, and could damage downstream components if shed.
\end{abstract}




\section{Fundamental Ice Crystal Accretion Physics Studies}

\section{Tom Currie, Danny Knezevici \\ Dan Fuleki}

National Research Council of Canada

Gas Turbine Laboratory, Icing Group

Peter M. Struk, Andy P. Broeren

Jen-ching Tsao, Mario Vargas, William Wright

NASA Glenn Research Center

Icing Branch

S4E든 International"

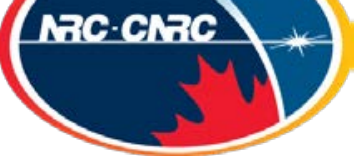




\section{Background}

- Ingestion of atmospheric ice crystals by aircraft engines can cause ice to accrete on internal components , leading to rollback, flameout, mechanical damage, etc.

- Ice crystal icing observed in experiments at NRC with flat plate, intercompressor S-duct (with strut), but understanding/verification of even basic physics still incomplete

- Improved understanding of physics required to develop simulation models and scaling laws/ parameters

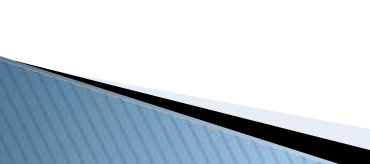




\section{Where Do Crystals Accrete?}

- Some melting of crystals required

- $\rightarrow$ favors accretion several stages into compressor, on non-rotating components

- Accretion most likely on surfaces with:

- cooling (e.g. by bypass air)

- anchoring geometry (e.g. probe, bleed port)

- low tangential velocity/ shear, high collection efficiency (stagnation points)

- Cannot be more precise without answering the question

Why Do Crystals Accrete?

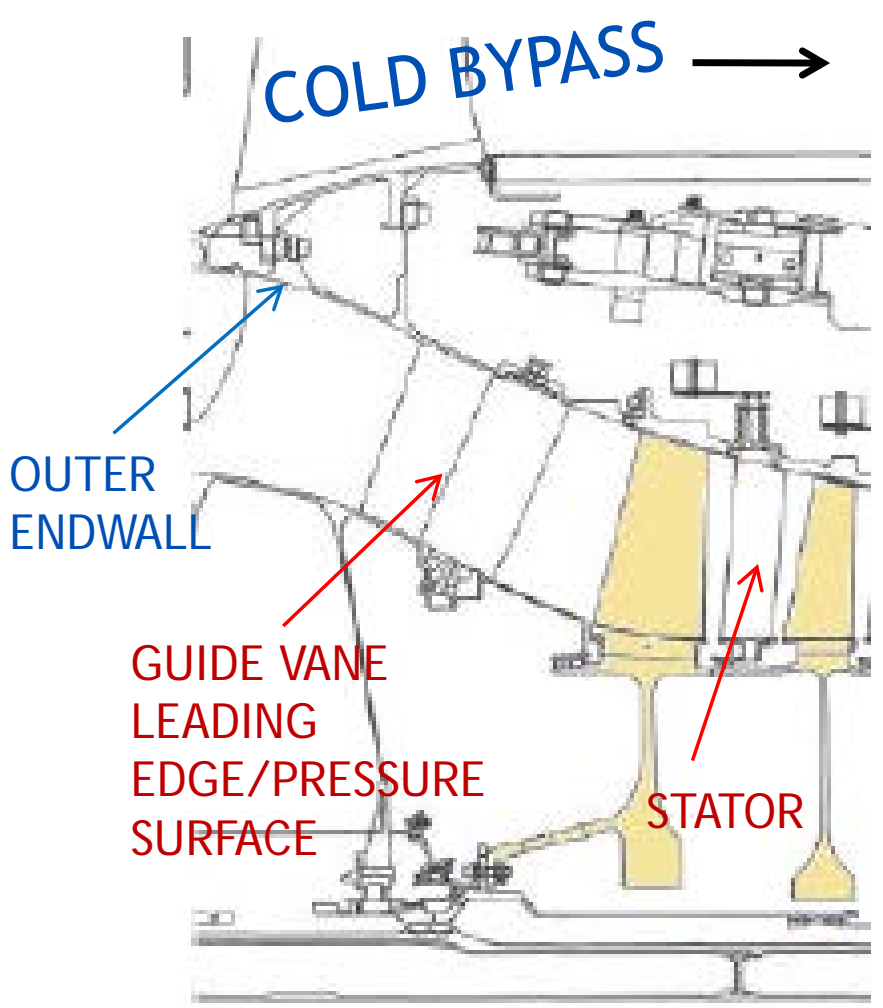

S4E International" 


\section{Research Objectives}

- Preliminary study of why crystals accrete

- conditions/ parameters governing accretion initiation and growth

- Determine accretion characteristics of a simple geometry (surface) over range of mixed-phase icing conditions

- Document time evolution of ice accretions - initiation, growth, shedding

- Obtain baseline heat transfer and aerodynamic characteristics for test geometry, without ice crystals or water spray

- No attempt to identify where crystals accrete - focus on identifying local parameters to be used with stage-by-stage calculations of aero-thermodynamic parameters, crystal melting, etc. to identify critical locations

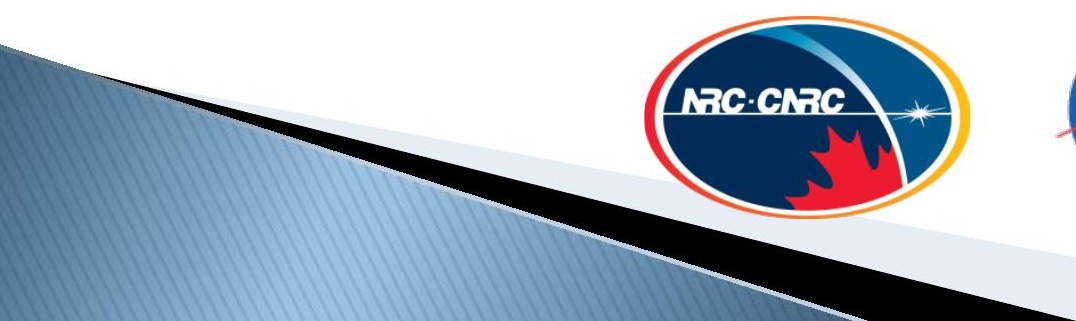

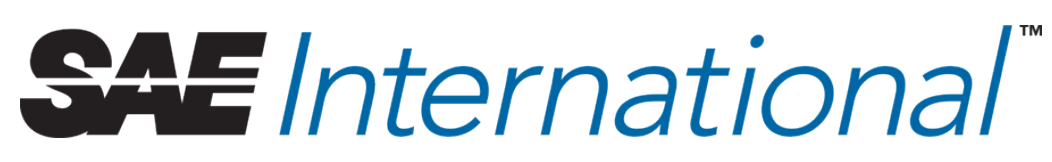




\section{Test Surface Design Objectives}

Allow high (closer to normal) particle incidence angles, as expected for a stator pressure surface

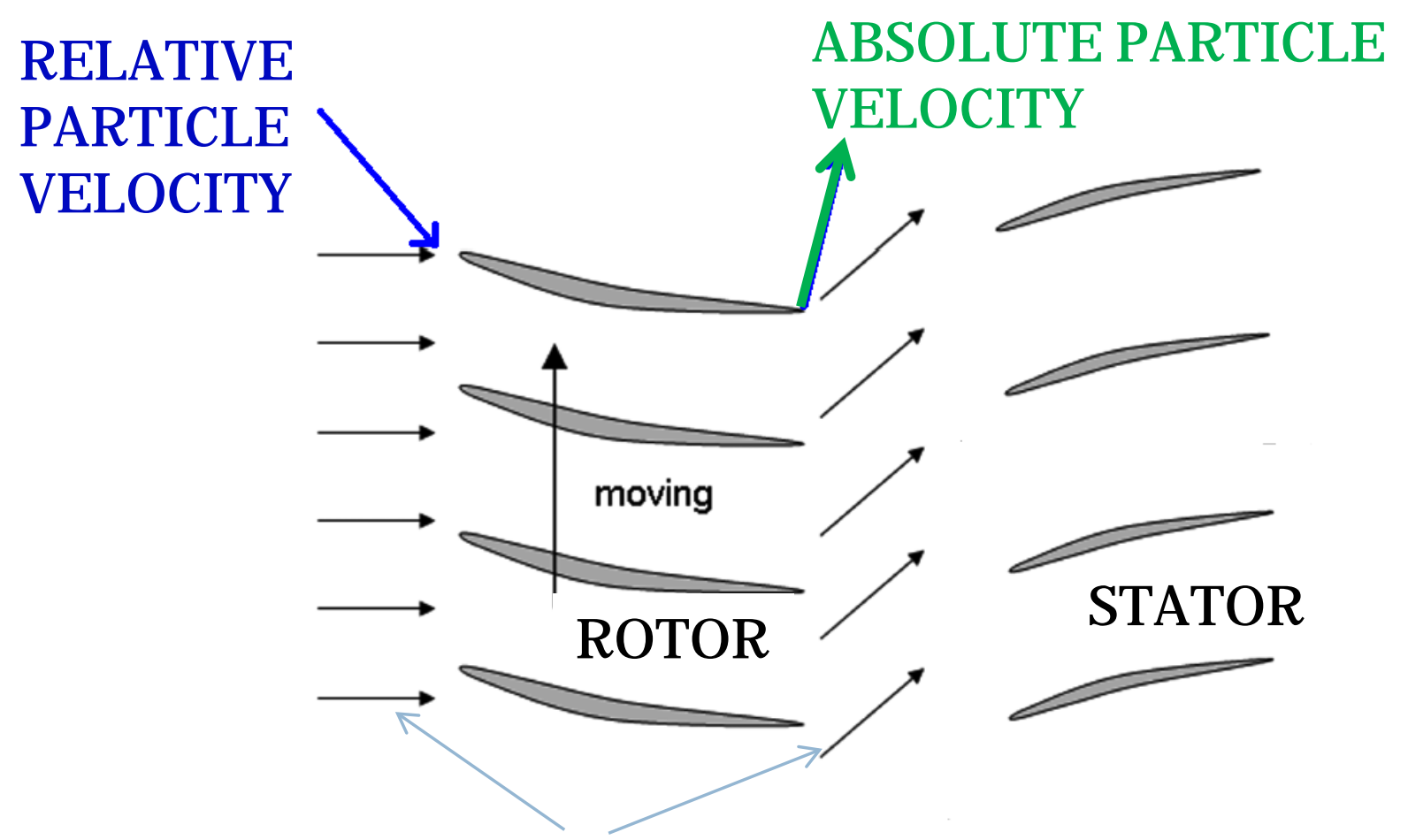

Absolute air velocities

SAcElinternational"

2011-38-0018

Fundamental Ice Crystal Physics Studies 


\section{Test Surface Design Objectives, cont'd}

1 Provide $\sim 2 \mathrm{D}$ flow

- Have relatively sharp leading edge, characteristic of compressor blades

- No flow separation on clean geometry

- Flat icing surface to facilitate mounting surface instrumentation, back side heaters, etc.

- Provide adequate space behind test surface to allow attachment of heaters, compact transducers, etc

- Similar thermal boundary conditions to hollow Ti 6Al-4V compressor blade

- Material other than test surface ideally ice-phobic with low thermal conductivity

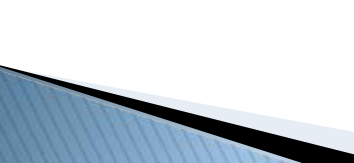




\section{Test Surface Design Objectives, cont’d}

- Pressure surface of wedge airfoil forebody satisfied requirements
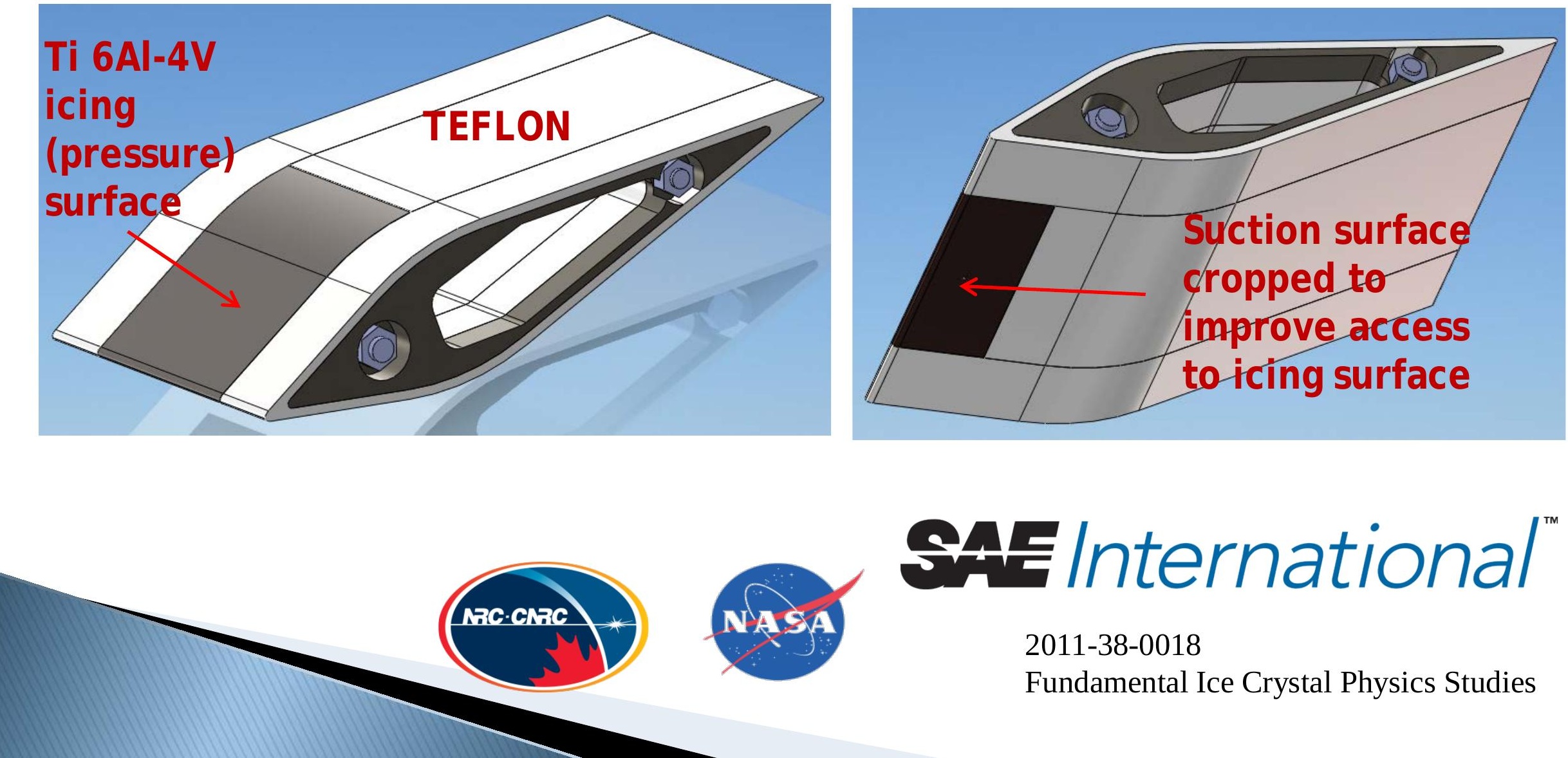

S4F International"

2011-38-0018

Fundamental Ice Crystal Physics Studies 


\section{Wedge Airfoil Geometric Parameters}

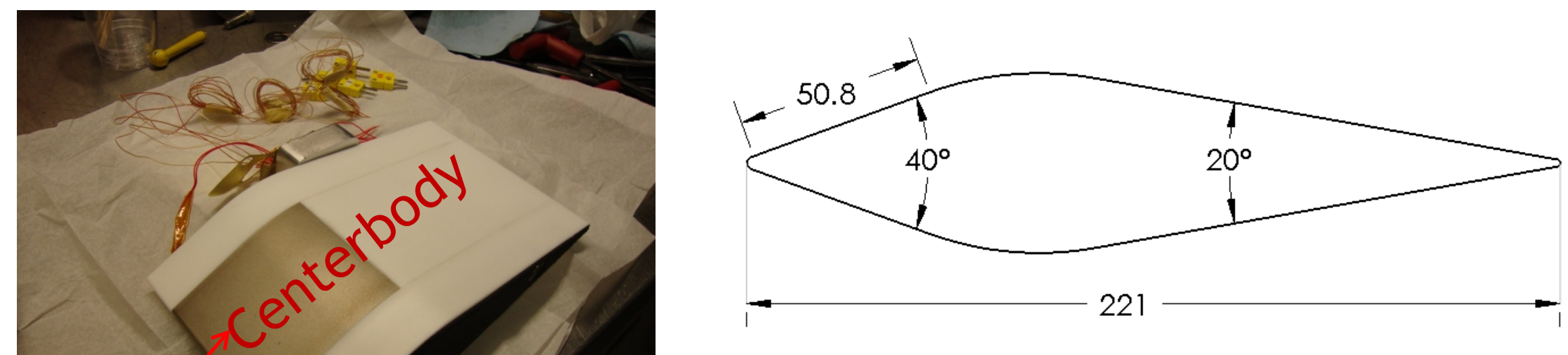

- Symmetrical

Ti insert

- chord =221mm (8.7")

- max thickness $=22.2 \%$ chord

- $50.8 \mathrm{~mm}$ (2") x $3.175 \mathrm{~mm}$ (1/ 8" thick) flat icing surface

\section{SA든 International" 2011-38-0018 \\ Fundamental Ice Crystal Physics Studies}




\section{NRC Test Facility}

- Tests conducted in an icing cascade tunnel located in NRC Research Altitude Test Facility (RATFac) altitude chamber.

Rectangular inlet bellmouth

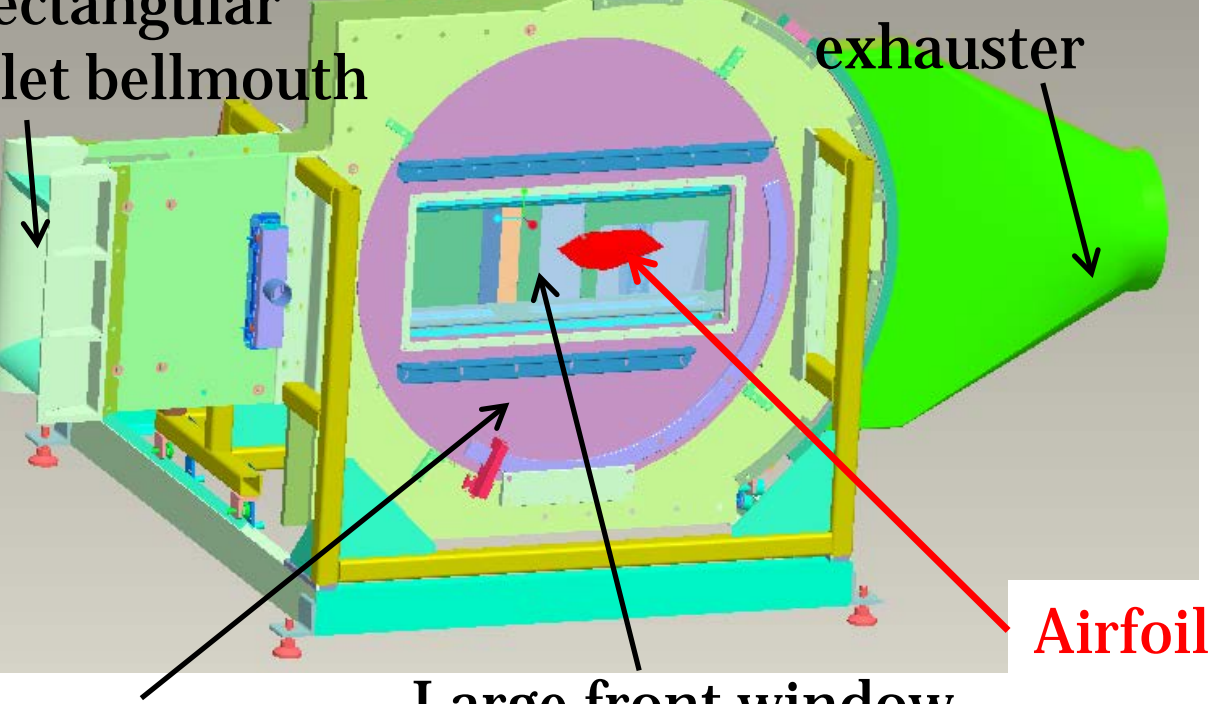

Turntable (not used)
Fixed heated $\mathrm{p}_{\mathrm{o}}$ Traversed cobra/ $\mathrm{T}_{\mathrm{o}}$

\section{Connection to $\mathrm{T}_{\mathrm{o}}$ probe}

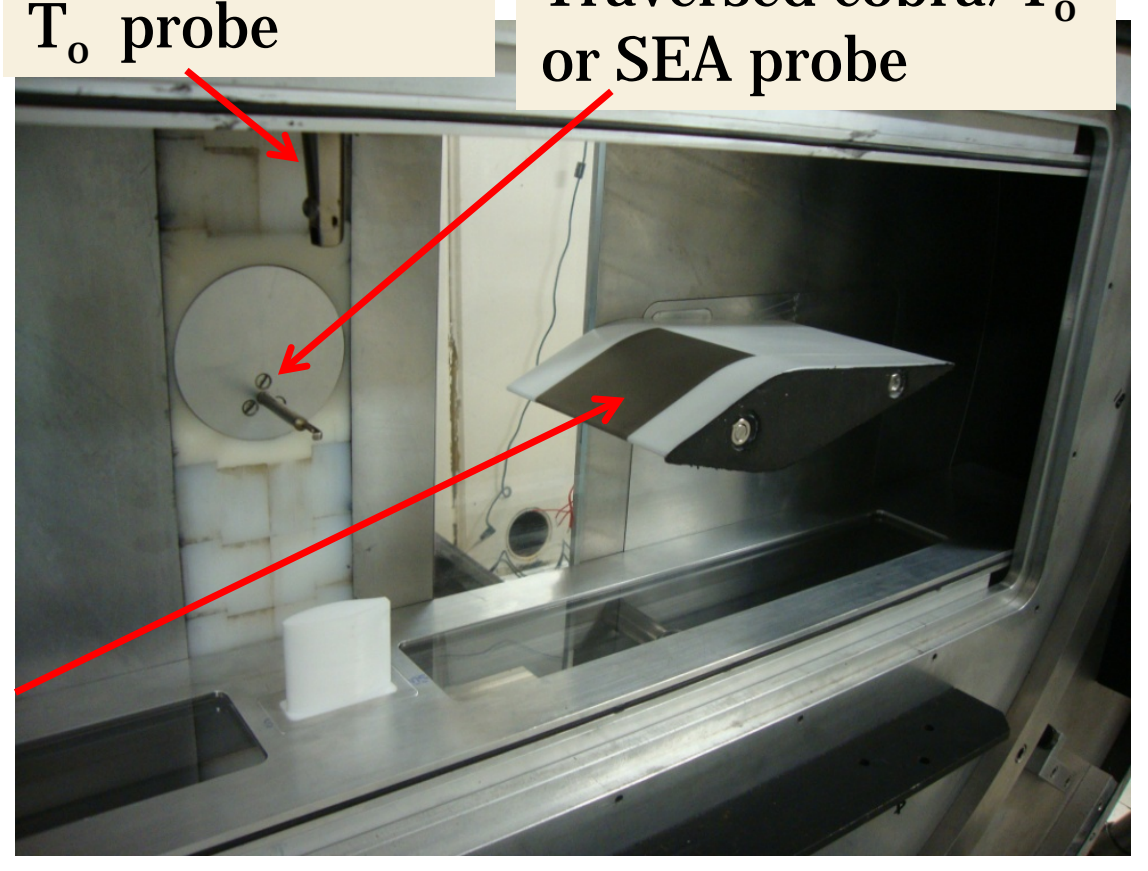

노들 International"

2011-38-0018

Fundamental Ice Crystal Physics Studies 
NRC Test Facility, cont'd

Supplementary water spray nozzles

Cold jet with ice

Warm air from chamber
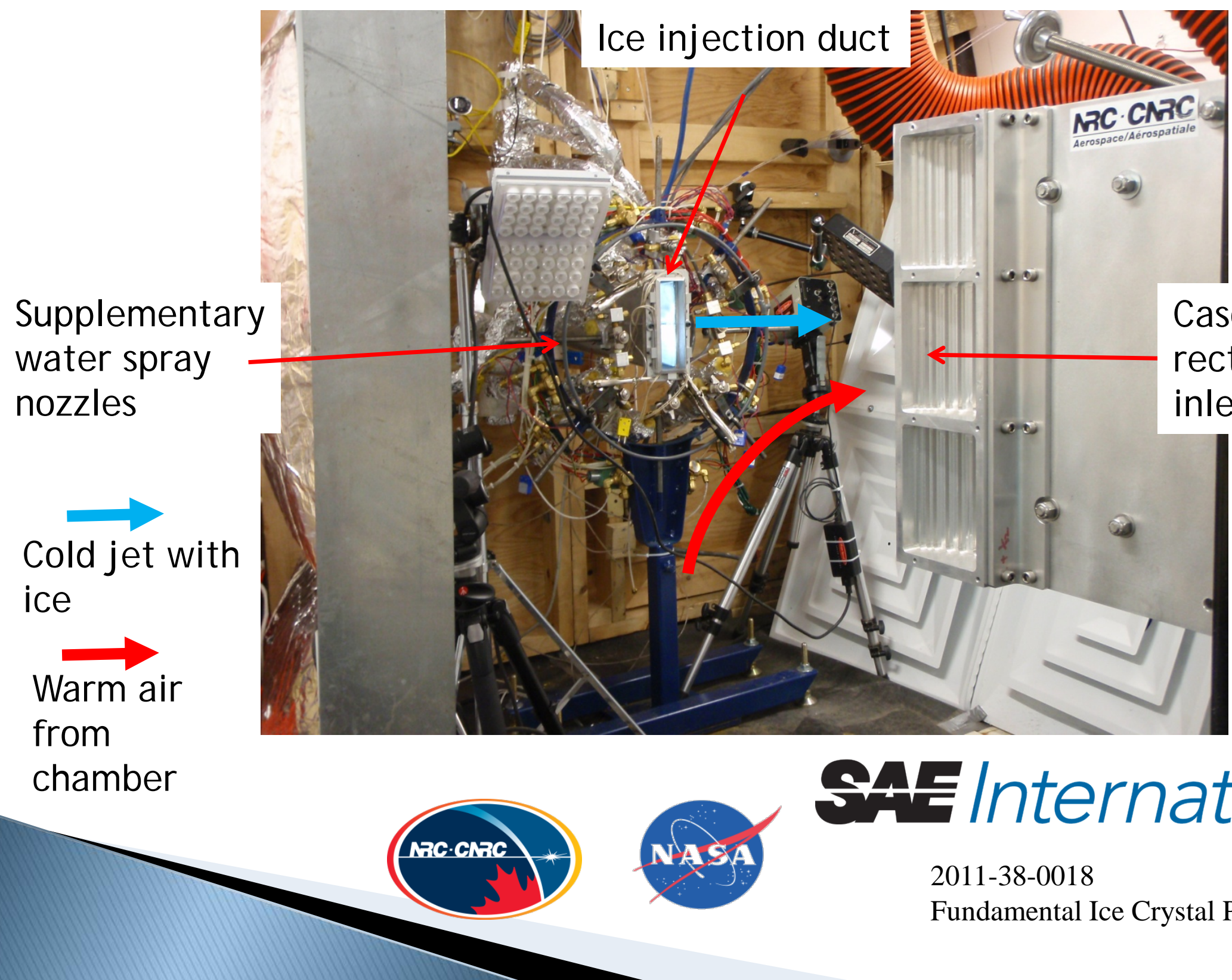

S4F International"

2011-38-0018

Fundamental Ice Crystal Physics Studies 


\section{Baseline (Clean Airfoil) Aerodynamic Tests}

- Static pressure measurements to determine isentropic Mach number distribution and oil flow tests to check for flow separation

- Aluminum centerbody with $42 \quad 1 / 2 \mathrm{~mm}$ diameter static pressure taps at mid span replaced Ti insert

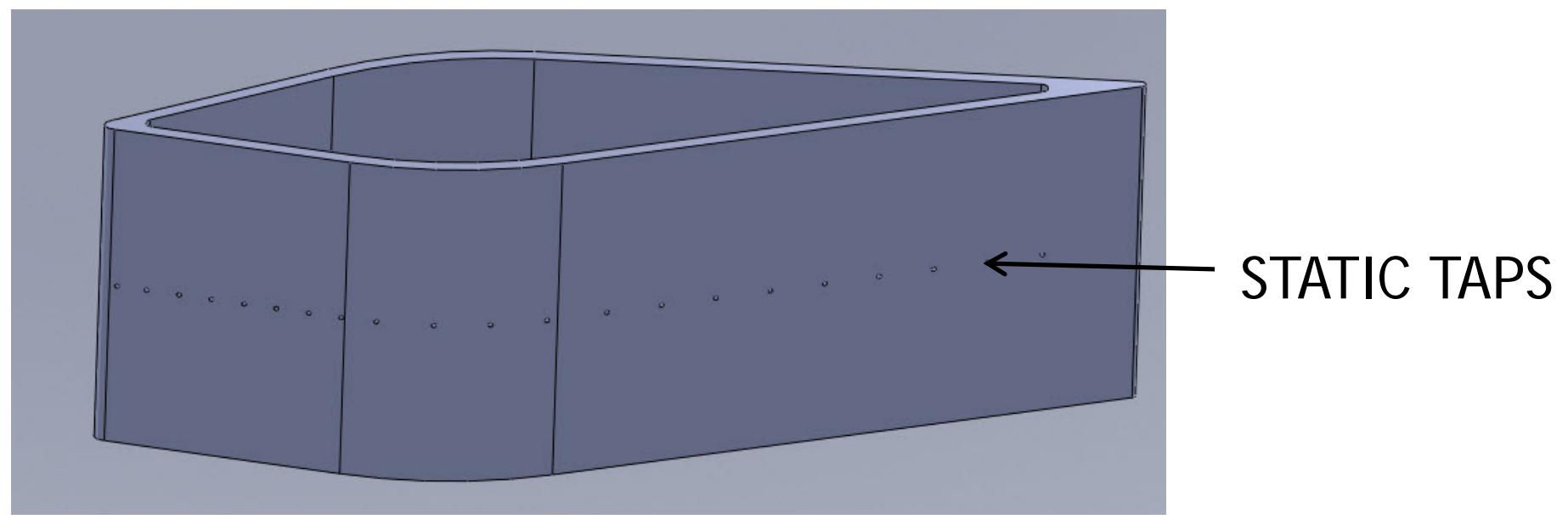

- Determined airfoil largely separation-free over required operating range, and flow over forebody pressure (icing) surface well-behaved

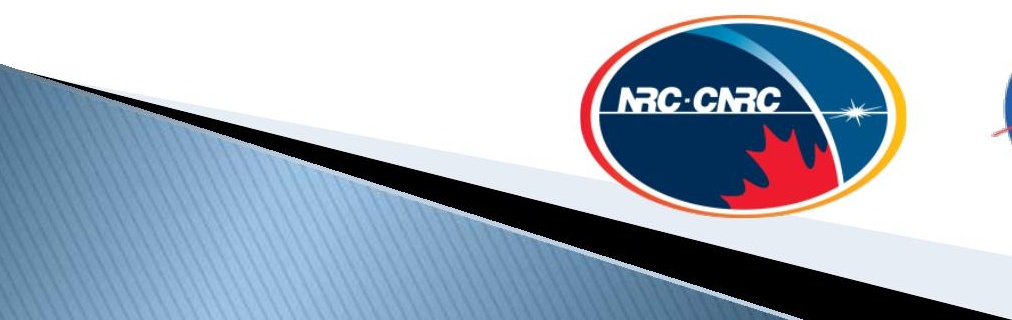

s4든 International 


\section{Heat Transfer Tests}

- Ti insert replaced with copper, heated with thin film heaters

- 5 type $\mathrm{K}$ thermocouples embedded in copper wall

- 4 thin film heat flux gauges on insert pressure surface

- $\mathrm{T}_{\mathrm{o}}, \mathrm{M}$, turbulence parameters measured $150 \mathrm{~mm}$ before leading edge

- Effect of gauge on heat flux corrected with $\mathrm{CFD} \rightarrow q^{\prime \prime} \rightarrow h=\frac{T_{\text {wall }}-T_{\text {recovery }}}{q^{\prime \prime}}$
- Tested at $-6^{\circ} \mathrm{AOA}, 45 \mathrm{kPa}$ (6.5psia)
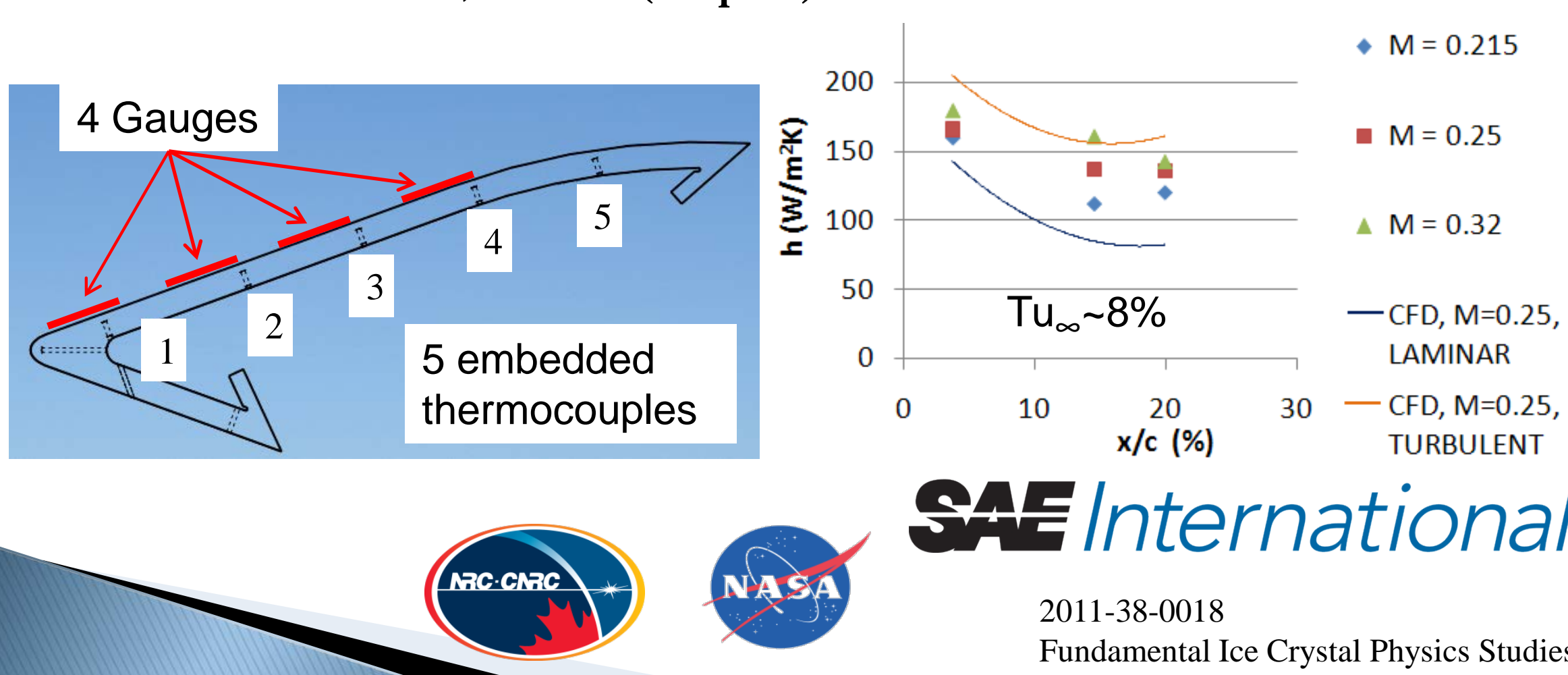

SA든 International" 


\section{Icing Tests}

Controlled Parameters

- $\mathrm{M}=0.2$ (most tests) and 0.3

- $\mathrm{P}_{\mathrm{o}}=45$ or $93 \mathrm{kPa}(6.5,13.5$ psia), most tests

- Total air temperature (TAT) above freezing $\left(5 \rightarrow 15^{\circ} \mathrm{C}\right)$

- Injected IWC up to $20 \mathrm{~g} / \mathrm{m}^{3}$ (bulk) $\rightarrow \mathrm{IWC}_{\mathrm{i}}$

- Injected (supplemental) LWC up to $3 \mathrm{~g} / \mathrm{m}^{3} \rightarrow \mathrm{LWC}_{\mathrm{i}}$

- Angle of attack $=-6^{\circ}$ (nose down)

- MMD of ice particles fixed at $\sim 100-200 \mu \mathrm{m}$

- MVD of water droplets $=40 \mu \mathrm{m}$ (sea level calibration)

Parameters Measured or Calculated

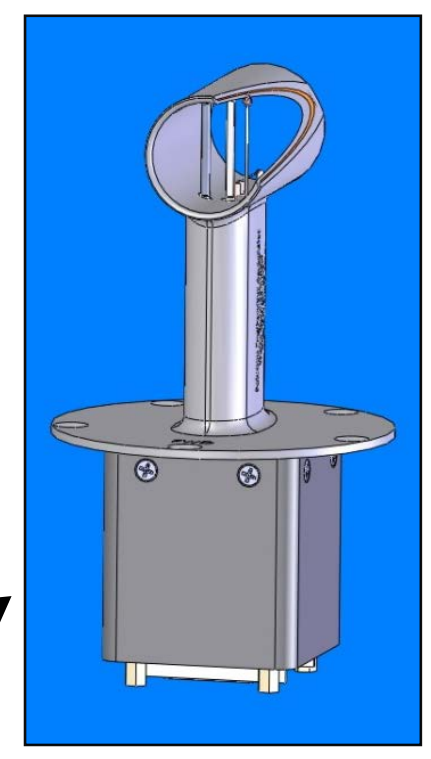

v IWC, LWC measured with SEA multi-element probe $150 \mathrm{~mm}$ upstream of airfoil $\rightarrow \mathrm{IWC}_{\mathrm{m}}, \mathrm{LWC}_{\mathrm{m}}$

- Wet bulb temperature TWB, calculated from mass balance on moisture entering cascade tunnel from altitude chamber (ambient) and ice injection pipe (dehumidified).

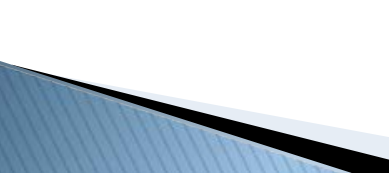




\section{Icing Tests}

Parameters Measured or Calculated, cont'd

- Temperatures of icing surface, measured with 5 type K thermocouples

- Icing recorded with video/ still cameras from 3 angles, front (perspective below) , side, top

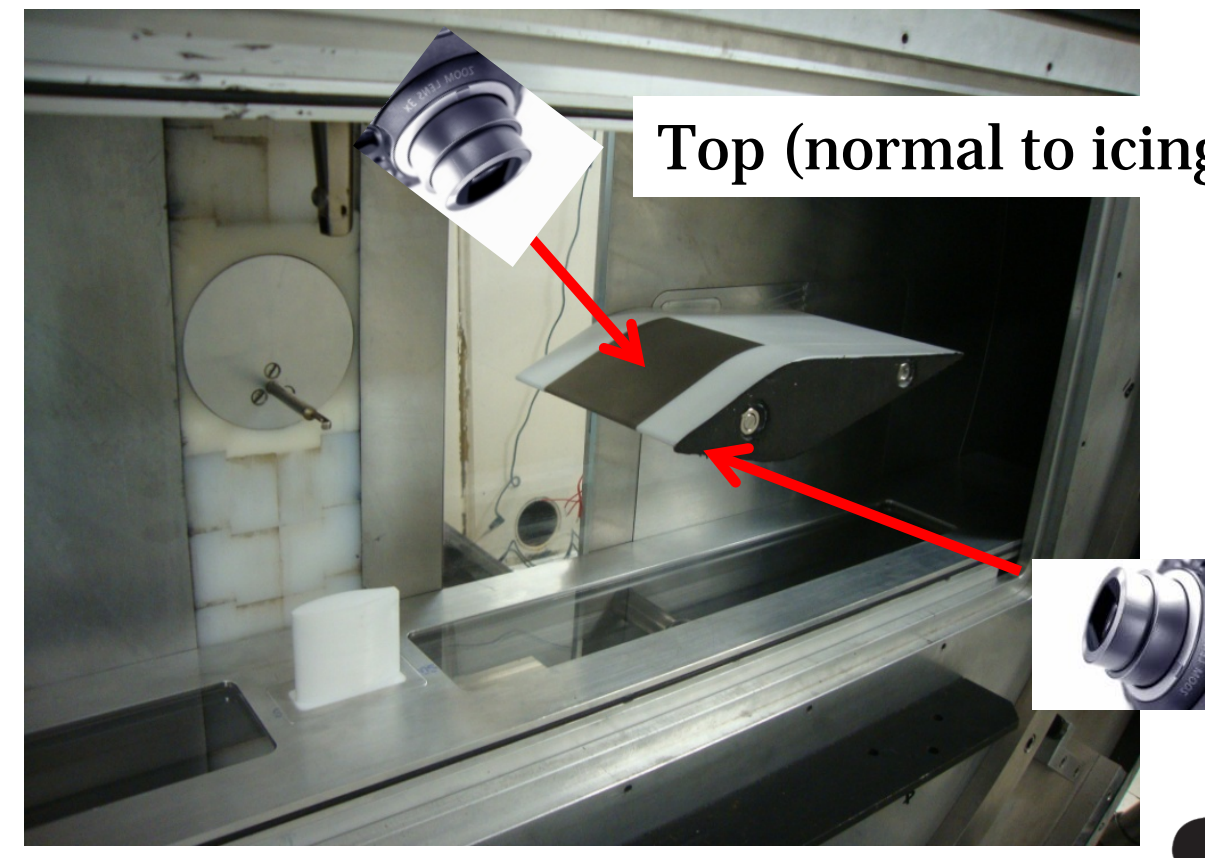

Side (end view of leading edge)

SAEI International"

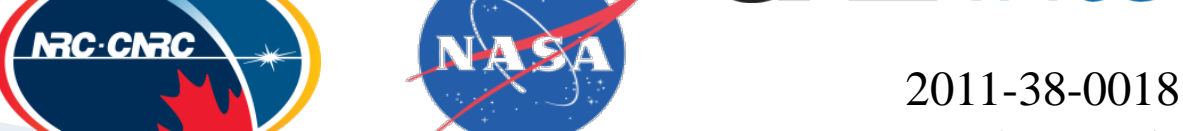

Fundamental Ice Crystal Physics Studies 


\section{Imaging Views}

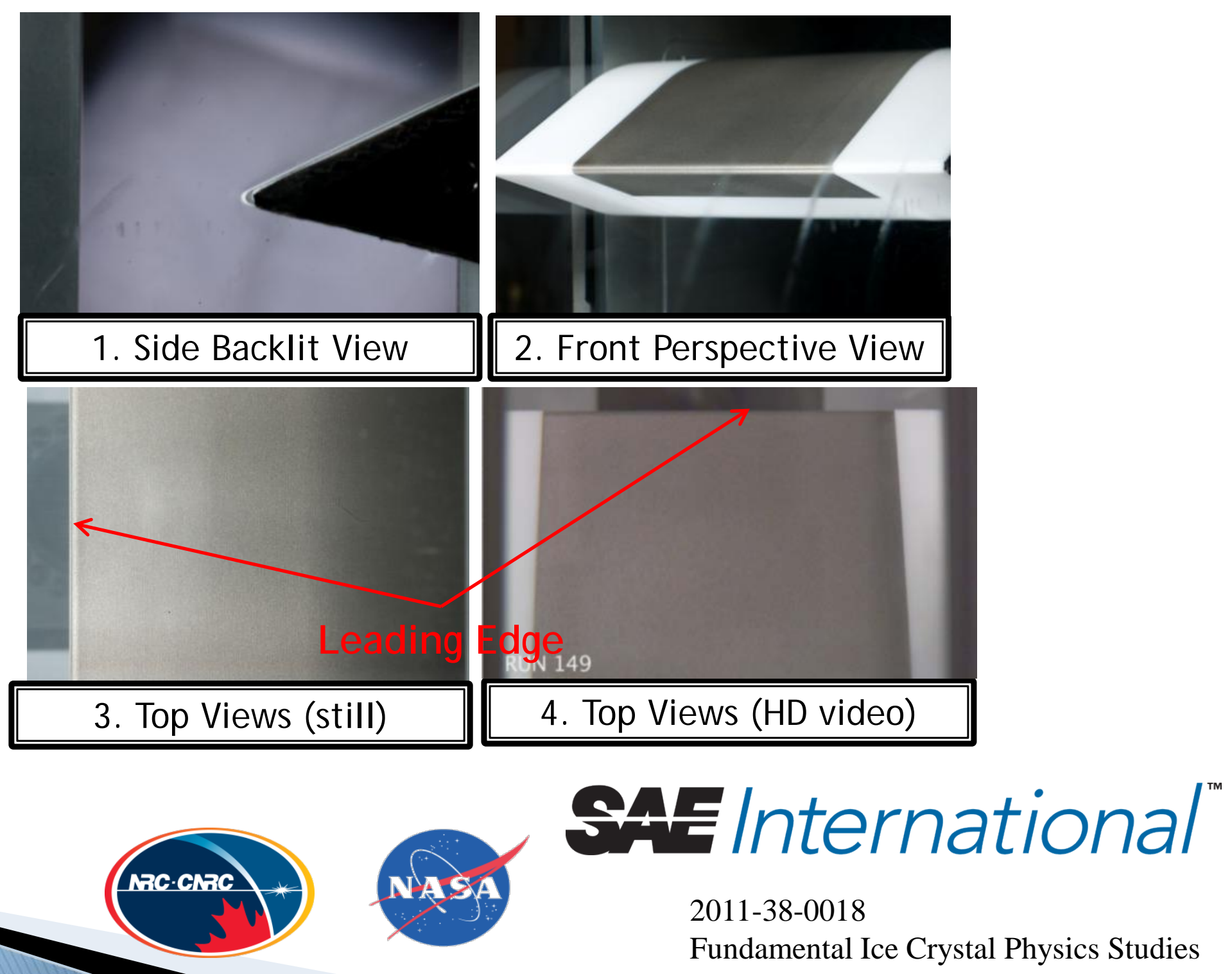




\section{Icing Test Procedure}

- Tunnel operating conditions adjusted to obtain desired (dry air) test section $\mathrm{p}_{0}, \mathrm{TAT}, \mathrm{M}$

- Multi-element probe used to determine spray water flow for supplemental LWC

- Steady-state data recorded under dry conditions

- Ice and water flows started. Ice mass flow for required IWC $\left(\mathrm{g} / \mathrm{m}^{3}\right)$ determined from tunnel air volume flow.

- Data and video images recorded continuously during 3-10 minute test

- SEA probe inserted at end of test to measure IWC and LWC

- Airfoil deiced for next test

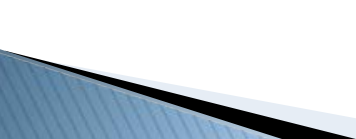




\section{Icing Test Results}

- Most tests performed at $45 \mathrm{kPa}$ or $93 \mathrm{kPa}(6.5,13.5 \mathrm{psia}), \mathrm{M}=0.2$

- TAT typically $>6^{\circ} \mathrm{C}$ higher at $45 \mathrm{kPa}$ than at $93 \mathrm{kPa}$, but accretions generally much larger and better adhered at lower pressure

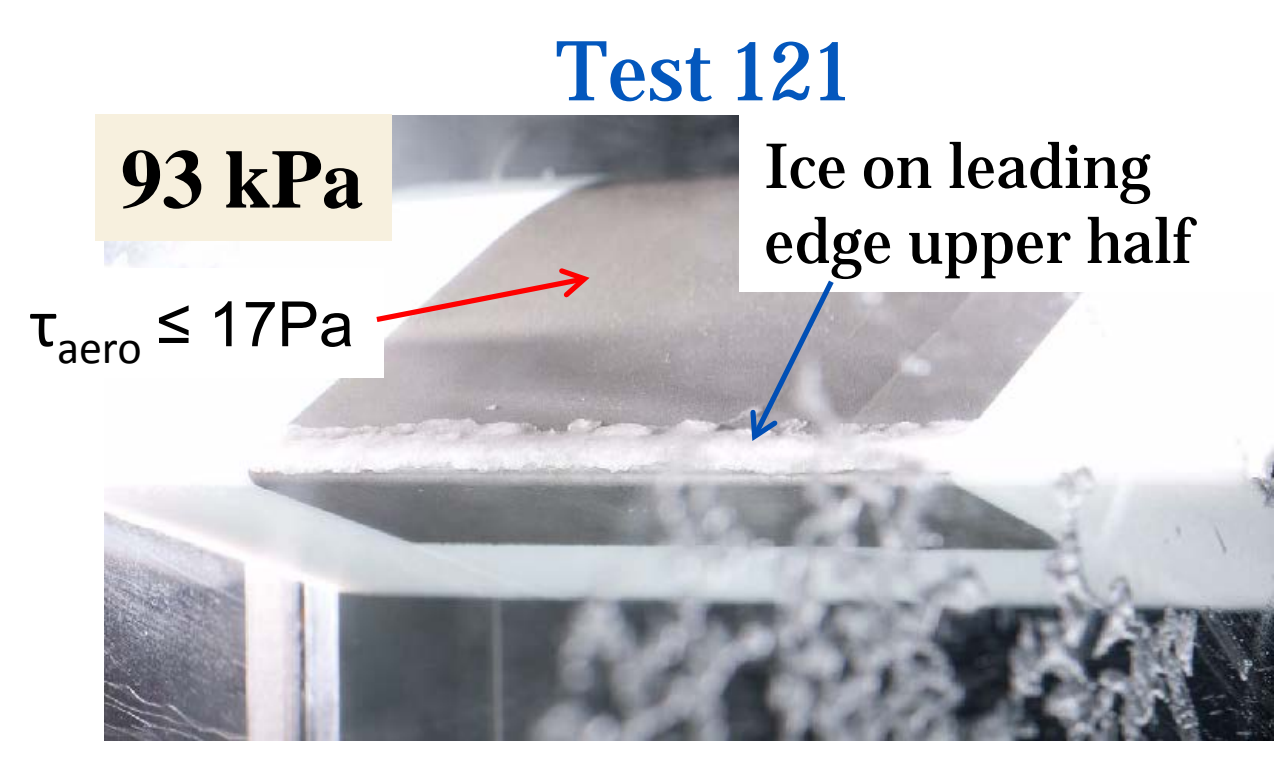

$\mathrm{TAT}=\mathbf{6 . 2} \mathbf{2}^{\circ} \mathbf{C}, \mathrm{LWC}_{\mathrm{i}}, \mathrm{IWC}_{\mathrm{i}}=0,20 \mathrm{~g} / \mathrm{m}^{3}$ $\mathrm{LWC}_{\mathrm{m}} / \mathrm{TWC}_{\mathrm{m}}=20 \%$

(note: SEA underpredicts LWC, TWC)
Test 149

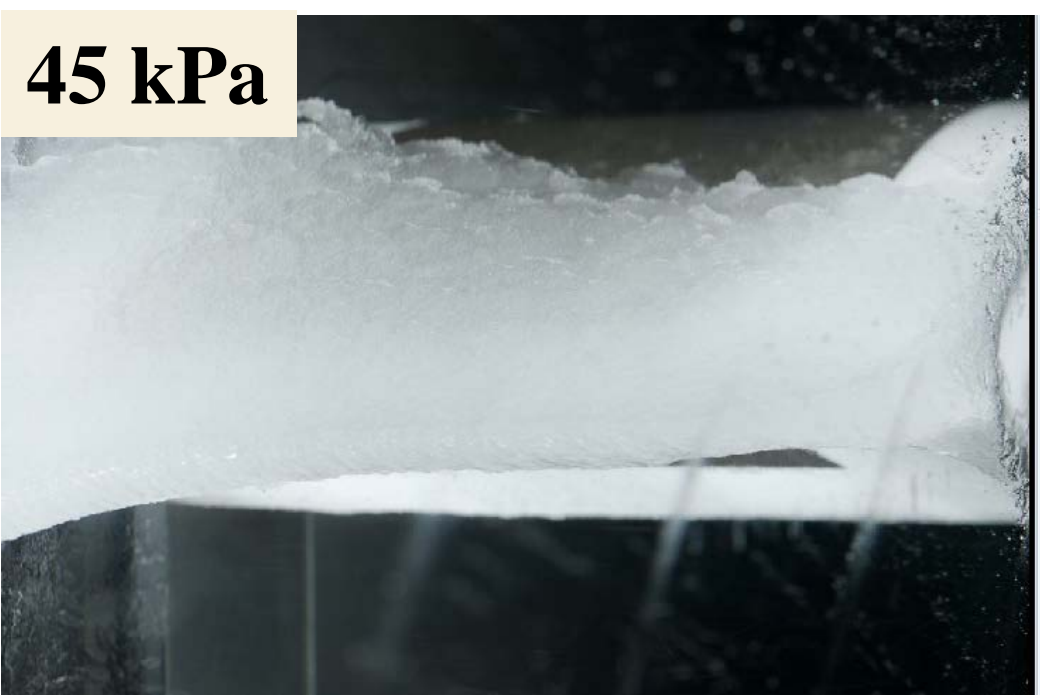

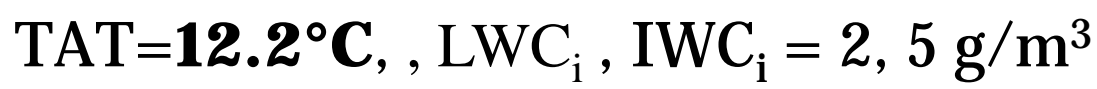
$\mathrm{LWC}_{\mathrm{m}} / \mathrm{TWC}_{\mathrm{m}}=42 \%$

SAE International" 


\section{Icing Test Results, cont'd \\ Why the Strong Dependence on Pressure?}

- Believed to be due primarily to stronger evaporative cooling at lower pressure, characterized by wet bulb temperature TWB

- At TWB $\{=\mathrm{f}(\mathrm{T}, \mathrm{p}$, moisture content $\omega)\}$

$$
\mathbf{q}_{\text {convection }}=\mathbf{q}_{\text {evaporation }} \quad \text { (make-up water at TWB) }
$$

for wet adiabatic surface (closely approximated by hollow Ti blade)

- Evaporation also cools impinging water

- Melting of ice helps lower surface temperature to $0^{\circ} \mathrm{C}$, but evaporation is required to lower to $0-\varepsilon$, needed for strong adhesion

- TWB ${ }_{0}$ based on $T_{0}, p_{0}$ is highest value on blade surface, so freezing possible everywhere when TWB ${ }_{0}<0^{\circ} \mathrm{C}$ depending on thermodynamics

- TWB $_{\text {o typically }}>0^{\circ} \mathbf{C}$ at $93 \mathbf{~ k P a}$ and $<0^{\circ} \mathrm{C}$ at $45 \mathrm{kPa}\left(\mathrm{TWB}_{\mathrm{o}}=3.7^{\circ} \mathrm{C}\right.$ in test 121 at $93 \mathrm{kPa},-3.7^{\circ} \mathrm{C}$ in test 149 at $45 \mathrm{kPa}$ )

- At $\mathrm{TWB}_{\mathrm{o}}<0^{\circ} \mathrm{C}$, impinging water froze on surface without added ice particles, similar to glaze ice. 


\section{Icing Test Results, cont'd}

Accretions with Water Only, and Water+Ice Crystals, $\mathrm{TWB}_{\mathrm{o}}=-3.7^{\circ} \mathrm{C}$

Test $151 \rightarrow 5$ min @ LWC $_{\mathrm{i}}=2 \mathrm{~g} / \mathrm{m}^{3}$ (water only)

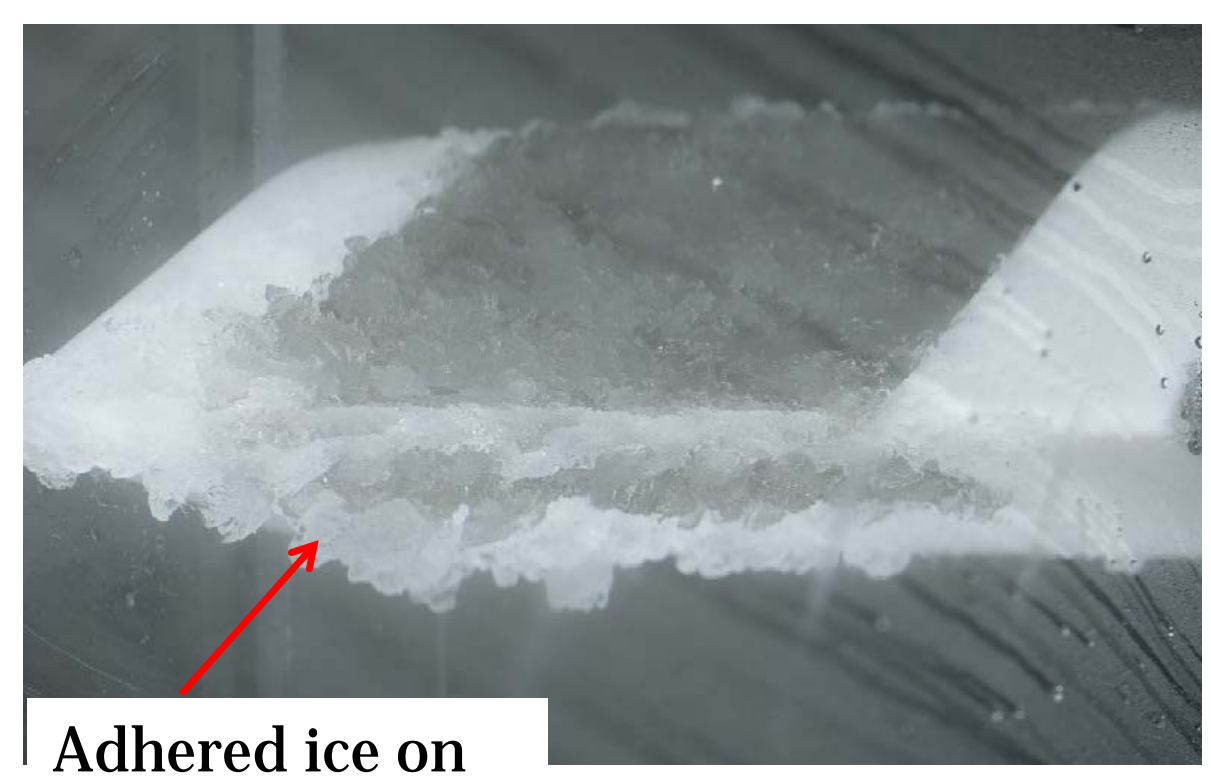
all Ti surfaces

$$
\mathrm{TAT}=13.4^{\circ} \mathrm{C}, 45 \mathrm{kPa} \mathrm{M}=0.21
$$

Test $151 \rightarrow 5 \mathrm{~min} @ \mathrm{LWC}_{\mathrm{i}}=2 \mathrm{~g} / \mathrm{m}^{3}$ $+1.5 \mathrm{~min} \mathrm{IWC}_{\mathrm{i}}=5 \mathrm{~g} / \mathrm{m}^{3}$

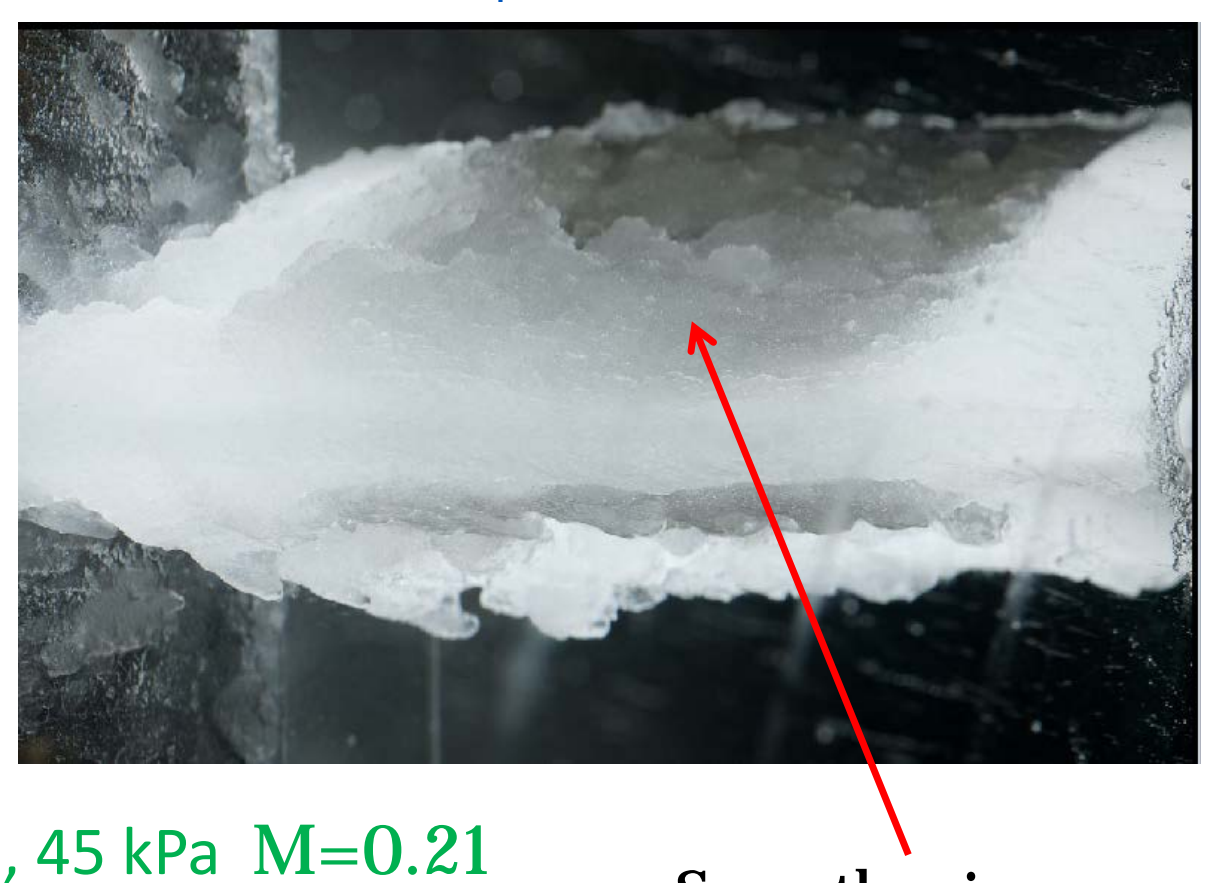

Smoother ice

-Effect of added ice greatest at leading edge

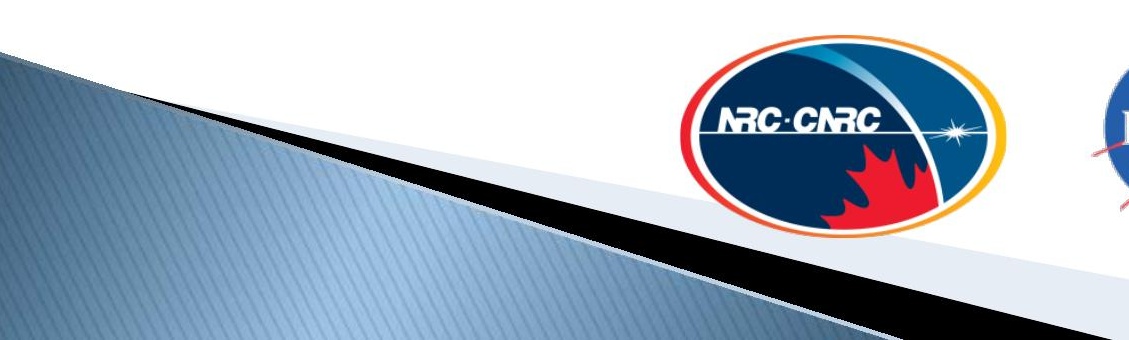

S4E International"

2011-38-0018

Fundamental Ice Crystal Physics Studies 


\section{Icing Test Results, cont"d}

Effect of Added Ice Crystals on Leading Edge Growth Rate

Test $151 \rightarrow 5$ min @ $\mathrm{LWC}_{\mathrm{i}}=$ $2 \mathrm{~g} / \mathrm{m}^{3}$ (water only) - top view
Test $151 \rightarrow 5$ min @ $\mathrm{LWC}_{\mathrm{i}}=$ $2 \mathrm{~g} / \mathrm{m}^{3}+1 \mathrm{~min} \mathrm{IWC}_{\mathrm{i}}=5 \mathrm{~g} / \mathrm{m}^{3}$
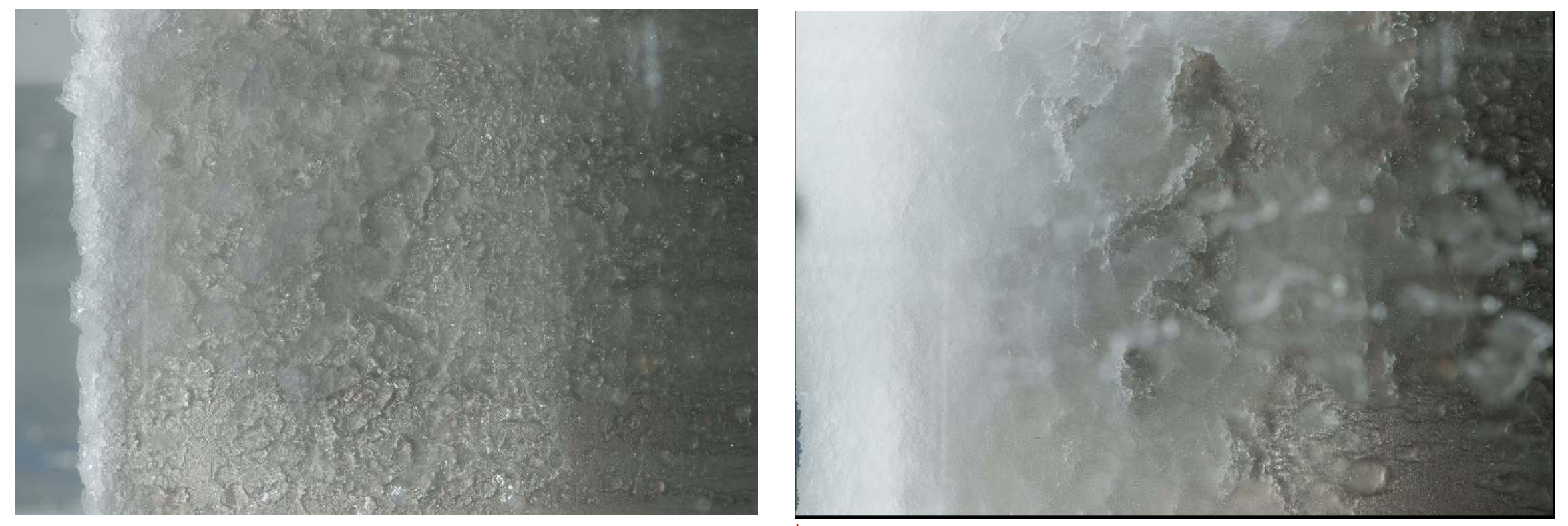

$\longleftarrow \sim 1 / 6 "$ leading edge growth

$>1 / 2$ " leading edge growth

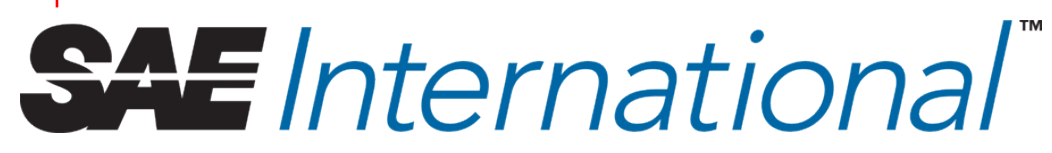

2011-38-0018

Fundamental Ice Crystal Physics Studies 


\section{Ice Thickness Measurement vs. Time - Test 151}

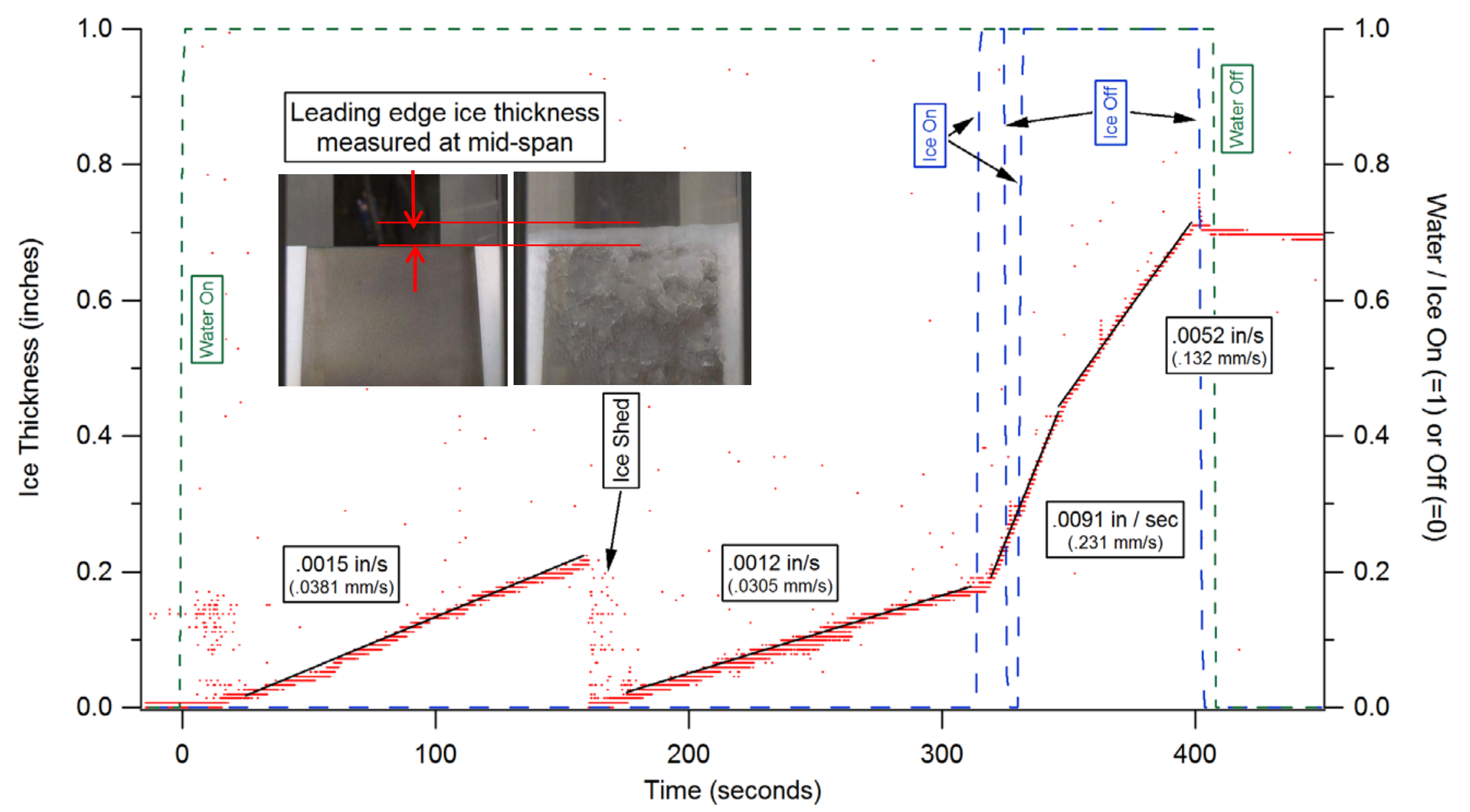

SA든 International" 


\section{Ice Thickness Measurement vs. Time - Test 149}

TAT $=12.2^{\circ} \mathrm{C}, 45 \mathrm{kPa}, \mathrm{LWC}_{\mathrm{i}}=2 \mathrm{~g} / \mathrm{m}^{3}, \mathrm{IWC}_{\mathrm{i}}=5 \mathrm{~g} / \mathrm{m}^{3}$

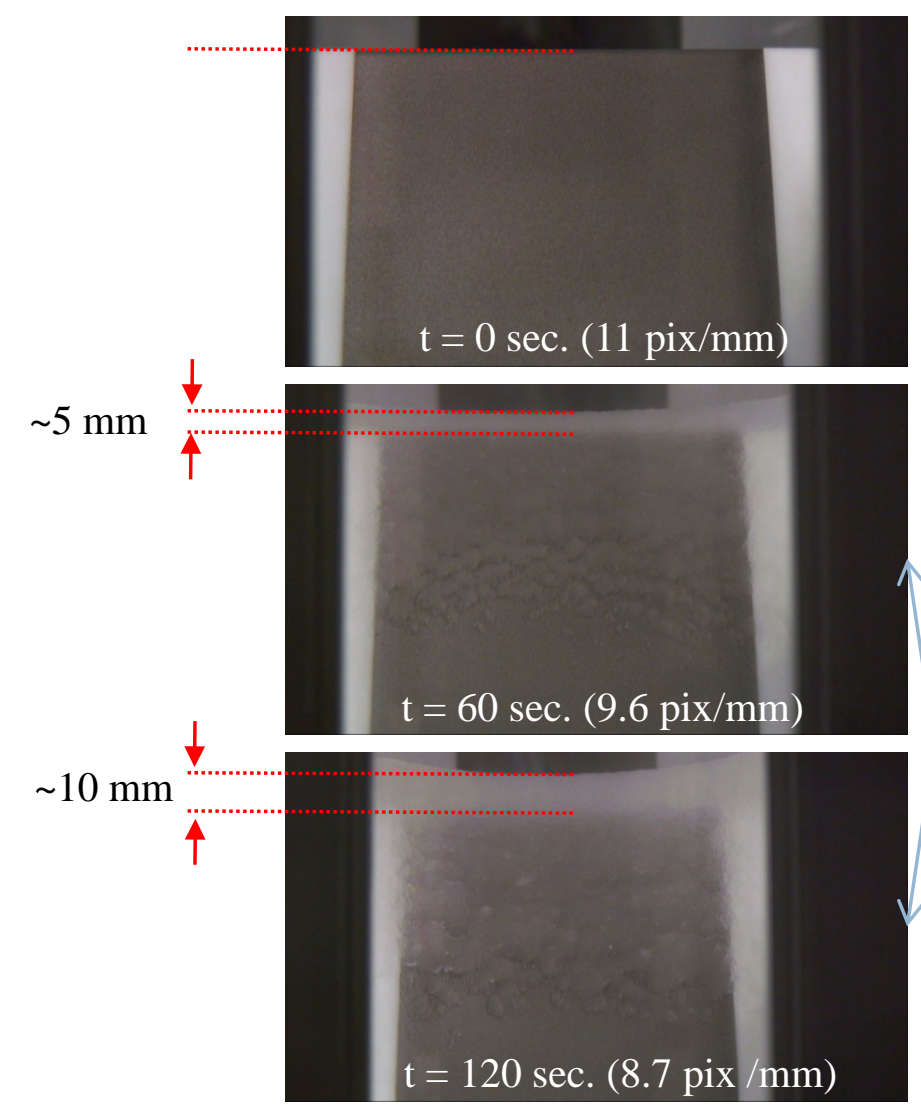

Zoom out for large ice growth

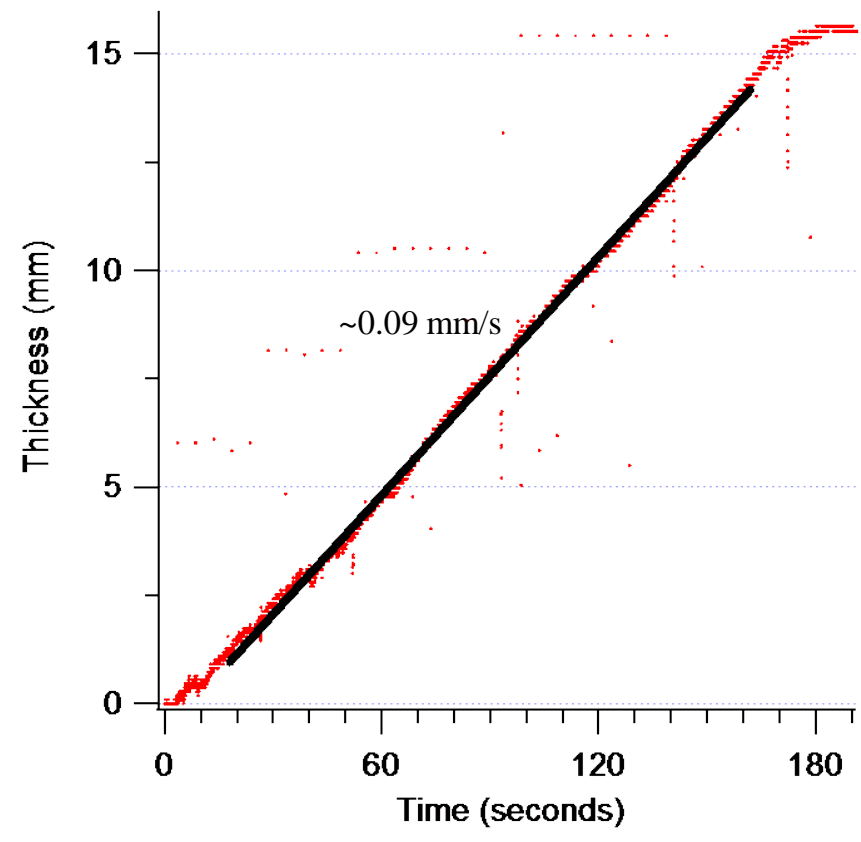

SA들 International"

Fundamental Ice Crystal Physics Studies 


\section{Icing Test Results, cont'd}

\section{Additional Experimental Observations}

- Significant natural melting of ice at 93kPa (LWC/ TWC from SEA $=20-32 \%$ ) so supplemental water usually not added.

- Insignificant natural melting of ice at 45kPa (LWC/TWC from SEA $=3-4 \%$ ) so only very thin accretion layers observed without adding supplemental water

- Teflon remained ice-free except at the leading edge unless ice crystals added, when freestream particles and ice sliding along endwalls accumulated at blade/ endwall junction

- Leading edge thermocouple $<0.5^{\circ} \mathrm{C}$ good indicator of whether ice would form 


\section{Conclusions}

- Results suggest strong evaporative cooling associated with TWB $<0^{\circ} \mathrm{C}$ provides mechanism for cooling surface exposed to impinging ice/ water + melted ice/ water below ice melting point, promoting freezing / $h i g h \tau_{\text {adh }}$

- Anchoring geometry (e.g. leading edge) generally required for significant accretion at TWB $>0^{\circ} \mathrm{C}$ because $\tau_{\text {adh }}$ is low

- Except near endwalls and/ or at low $\mathrm{M}\left(\tau_{\text {aero }}\right)$, serious accretions on stators/guide vanes only expected with TWB $<0^{\circ} \mathrm{C}$

- Additional experiments required to:

- confirm the significance of evaporative cooling (humidity control now available in RATFac)

- better understand the effects of pressure $\rightarrow$ altitude scaling

- explore the effect of Mach number variations on $\mathrm{TWB}_{\text {local }}$ (via effect on $p_{\text {static }}, \mathrm{T}_{\text {recovery }}$ ) and ice adhesion, erosion, etc.

- study geometries/ conditions allowing significant deposits of non- or loosely-adhered ice at TWB $>0^{\circ} \mathrm{C}$

S4든 International" 


\section{Acknowledgments}

- NRC authors would like to thank the FAA, Transport Canada, and NRC for their support and financial assistance

- NASA authors acknowledge support by the Atmospheric Environment Safety Technologies (AEST) project

- All authors would like to thank Tom Bond of the FAA for his guidance and support, and Chris Lynch for imaging support

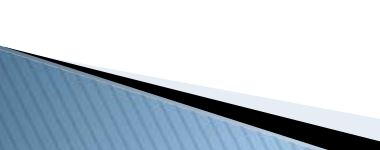




\section{Questions?}

SA든 International"

2011-38-0018

Fundamental Ice Crystal Physics Studies 